\title{
Clinical and Histological Studies in Diabetic Neuropathy
}

\author{
A Reassessment of Vascular Factors in Relation to Intravascular Coagulation \\ W. R. Timperley, J. D. Ward, F. E. Preston, T. Duckworth and B. C. O'Malley \\ Departments of Neuropathology, Medicine, Haematology and Orthopaedics, The Royal Infirmary, Sheffield, England
}

\begin{abstract}
Summary. Sural nerve biopsy was performed in twenty-four diabetic patients, with clinical and electrophysiological evidence of diabetic neuropathy. Material from an autopsy case was also examined. Vessels plugged with fibrin were seen within nerve in nine cases. In three cases fibrin was observed tracking into the vessel wall and in four, older thrombus was observed in vessels. Areas of necrosis in nerve bundles were seen in two of the latter. In two patients there had been a preceding episode of intravascular coagulation. Fibrin deposition within small vessels could well play a part in damaging the diabetic nerve and a disturbance of the balance between deposition and removal by fibrinolysis could explain phasic variation in the symptoms of neuropathy.
\end{abstract}

Key words: Diabetes, neuropathy, intravascular coagulation, diabetic neuropathy, fibrin.

The aetiology of diabetic neuropathy has been a matter of controversy for many years. Fagerberg [1] described "vascular lesions in the form of hyalinisation, calibre reduction and thickening of the wall which were more common in patients with diabetic neuropathy than in other groups". He attributed such changes to progressive degenerative disease and assumed that they were responsible for the structural damage to the myelin sheath in the diabetic. Subsequent nerve biopsy studies did not confirm the presence of significant vascular lesions [2-3] and it seemed that firm evidence for a "vascular aetiology" of diabetic neuropathy was lacking. Biochemical changes have been demonstrated in diabetic nerve [4-6] and it would seem possible that nerve damage might be related to these changes.
Evidence is now accumulating of the existence of abnormalities of control of the fibrinolytic and coagulation system in diabetics [7-9]. Recently Timperley et al. [10] demonstrated thrombosis in small vessels, leading to ischaemic lesions in the brain and a variety of other organs, in diabetic patients dying in ketoacidotic coma. During life there is a continual balance between the deposition of fibrin on the vascular endothelium and its clearance by fibrinolysis. Variations in the balance of these mechanisms could explain the phasic nature of the clinical symptoms of diabetic neuropathy. A biopsy study was therefore undertaken to examine small blood vessels in diabetic peripheral nerve and changes within vessels were compared with those in a sample of skin removed at the time of biopsy.

\section{Materials and Methods}

Twenty-four diabetic patients were selected for the study. Criteria for selection were based mainly on the presence of significant symptoms of neuropathy, physical signs in this condition being notoriously difficult to assess accurately. It is our view that symptoms are more important in the diagnosis of diabetic neuropathy than physical signs. $75 \%$ of patients had sensory symptoms consisting of numbness, tingling and paraesthesiae of the lower leg and feet with a high incidence of nocturnal muscular cramps and shooting or burning pains. The remaining $25 \%$ had mainly motor symptoms varying from profound to less severe muscular weakness. There was some overlap in symptoms. Wasting of the small muscles of the hand was common in both groups. Diminished reflexes in the legs, patchy sensory loss and loss of vibration sense occurred in the majority of patients. Twenty-one patients were male and of these twelve were impotent. 
We also report the nerve histology of a young, newly diagnosed diabetic female, who died in diabetic coma. It was felt important to include this case in the study since it demonstrated that small vessel occlusion in intraneural vessels could occur in the very early stages of diabetes before clinical symptoms of neuropathy have appeared.

The mean age of this group of patients was 56 years (range $30-78$ years).

Following full clinical examination and assessment, motor conduction velocity (M. C. V.) in metres per second $(\mathrm{m} / \mathrm{sec})$ was measured in the lateral popliteal, median and uInar nerves, using a Medelec M.S. 5 machine. A mean of three measurements was taken. There is a wide range of normal nerve conduction (lateral popliteal nerve, $40-57 \mathrm{~m} / \mathrm{sec}$ ), but in only two of the patients studied was the nerve conduction in this nerve above $40 \mathrm{~m} / \mathrm{sec}$. The mean M. C. V. for the 24 patients studied was $32.8 \mathrm{~m} / \mathrm{sec} \pm$ SEM 1.42 , contrasting with a mean for our clinic in nonneuropathic diabetics $(41.6 \pm$ SEM $1.09(\mathrm{n}=38)$ and non diabetics $(48.3 \pm$ SEM $0.73(n=39))$. This degree of slowing of the M.C.V. indicated the presence of segmental demyelination. The non-neuropathic and non-diabetic patients are included for comparison, but were not submitted to sural nerve biopsy as we wished to keep the number of biopsies to a minimum. Informed consent was obtained from patients submitted to nerve biopsy. There have been no problems following this procedure either at the time of biopsy of after 1 year follow up.

Under local anaesthesia the sural nerve was exposed behind the lateral malleolus. If the nerve was of sufficient thickness, which was usually the case, it was split and a $1.5-2.0 \mathrm{~cm}$ length was taken from one half, leaving the nerve in functional continuity. An ellipse of skin was taken from the edge of the biopsy incision. As a control group sural nerves were taken from nondiabetic autopsy patients of the same age and sex distribution, along with sural nerve biopsies from ten patients with uraemic neuropathy. The sural nerves were removed at autopsy within ten hours of death; although it is possible that some fibrin may be removed following death it is our experience, from the study of cases of disseminated intravascular coagulation, that there is no obvious loss of fibrin in small vessels, at least up to twenty-four hours after death. All ten patients with uraemic neuropathy were within the age range of the twenty-four diabetic patients selected for biopsy study. Eight were cases of chronic glomerulonephritis, one had bilateral polycystic kidneys and one had nephrosclerosis. These nerves were, at the time, being studied as part of another research project and it was felt that they would serve as useful fresh control material for the present study. All these nerves showed evidence of demyelination.

Immediately after removal fragments of nerve were fixed in $3 \%$ phosphate buffered glutaraldehyde. Approximately $2 \mathrm{~mm}$ of nerve were fixed in $10 \%$ neutral formalin and $3 \mathrm{~mm}$ fixed in $1 \%$ buffered osmium tetroxide for teased nerve fibre preparation. Nerve embedded in paraffin was serially sectioned and stained with haematoxylin and eosin (H. E.), Martius-Scarlet-Blue (M.S.B.) solochrome-cyanine for myelin and Masson's trichrome stain.

No details of the clinical state of the patient were known to the pathologist (W.R.T.) at the time of assessment. No attempt was made to carry out a double blind study as it was not thought justifiable to perform nerve biopsies on patients with no evidence of diabetic neuropathy.

\section{Results}

In all cases evidence of demyelination was seen in longitudinally teased nerve fibre preparations - a well established method of assessing the degree of demyelination in nerve. No attempt was made to quantitate these results, but in general the degree of demyelination correlated with the severity of the neuropathic symptoms. Demyelination was often noted to be patchy with groups of demyelinated or demyelinating fibres situated between groups of normal fibres.

Vessels plugged or lined with fibrin (Fig. 1) were seen in eight patients with symptoms of diabetic neuropathy and in the patient studied at autopsy. The latter was a girl aged 18 who had experienced thirst and polyuria for about one year, but had never consulted her doctor. One week prior to admission she developed small inguinal abscesses and two days before admission she became anorexic, breathless and disorientated. She was admitted in coma (blood sugar $\left.649 \mathrm{mg} / 100 \mathrm{ml}, \mathrm{HCO}_{3} 3 \mathrm{mEquiv} / 1\right)$. Inspite of intensive therapy with intravenous fluids, insulin and small quantities of sodium bicarbonate, following which her metabolic state became normal within 6 hours, she failed to recover consciousness and was noted to have marked neck stiffness. Lumbar puncture revealed heavy blood stained fluid and she died three days after admission.

In three of these nine cases fibrin and red cells were seen to be infiltrating the vessel wall (Figs. 2 and 3 ). Four specimens showed older thrombus in small vessels (Fig. 4) and in two of these areas of necrosis were seen within nerve bundles (Fig. 5). In one case clumps of granular material were seen within the 


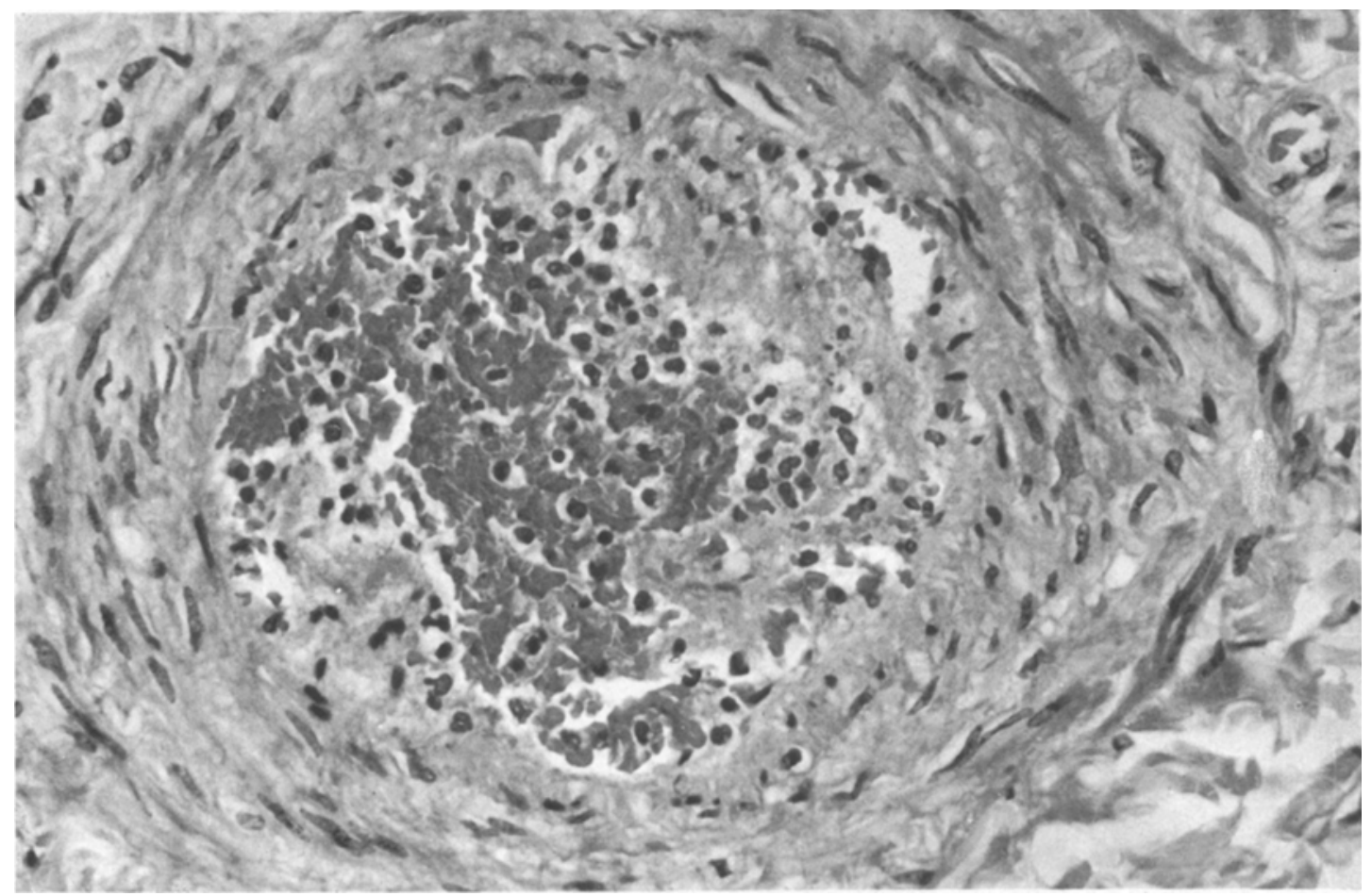

Fig. 1. Small perineural arteriole containing fibrin and red cells. HE stain $\times 600$

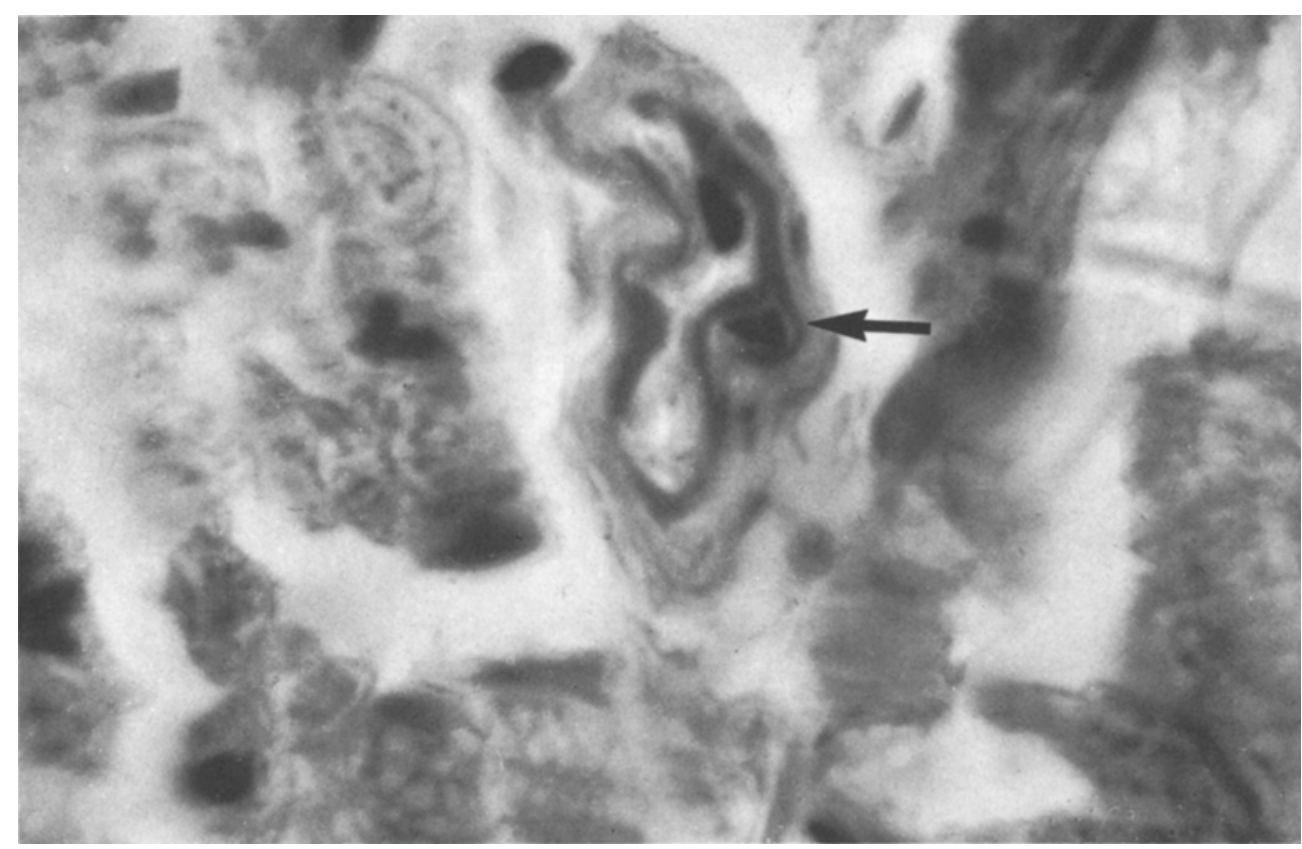

Fig. 2. Intraneural vessel lined by fibrin. Fibrin appears to be tracking into the vessel wall at one point (arrow). MSB stain $\times 2000$ 


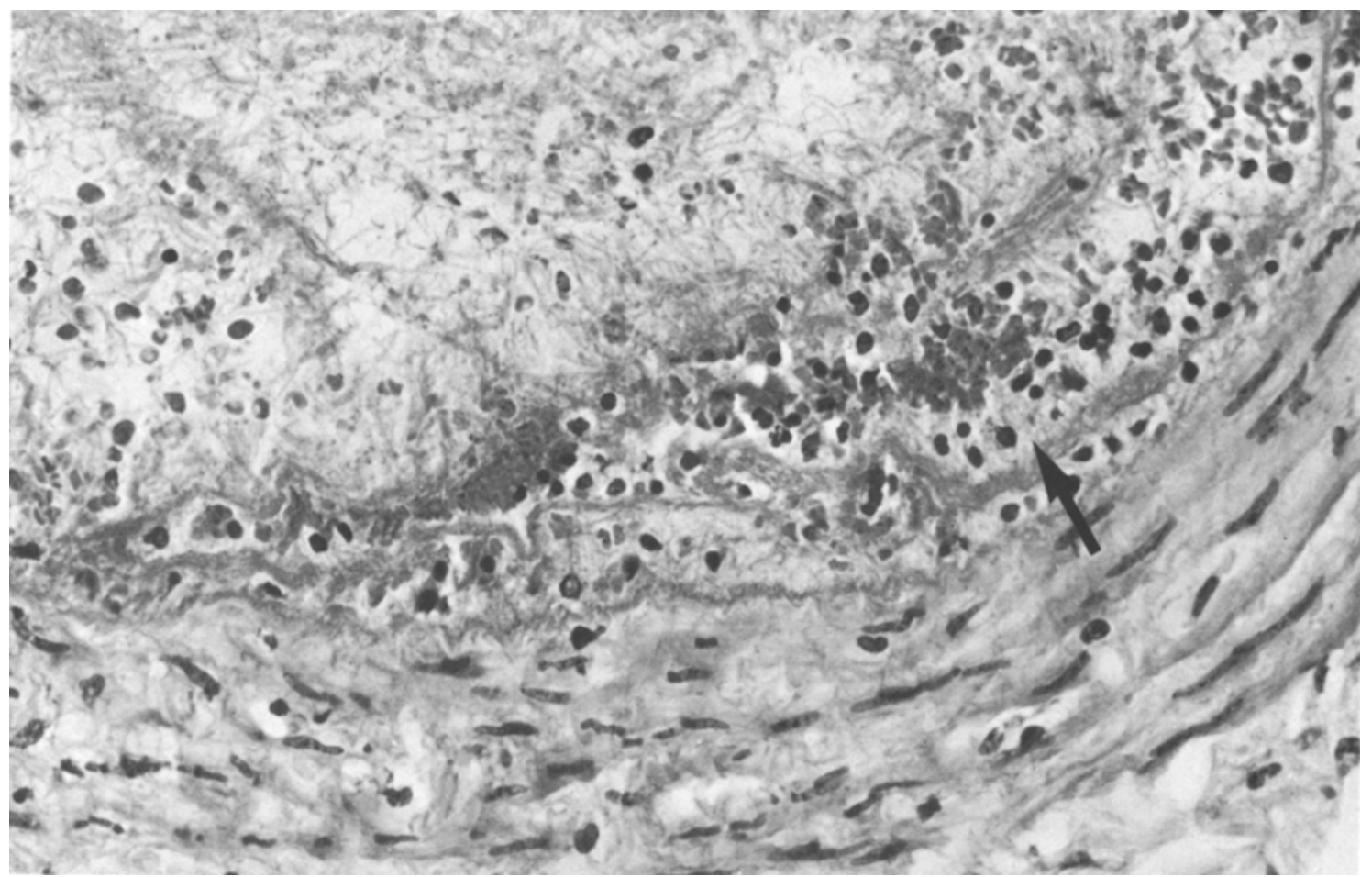

Fig. 3. Plasma and red cells tracking beneath the intima of a perineural vessel (arrow). HE stain $\times 960$

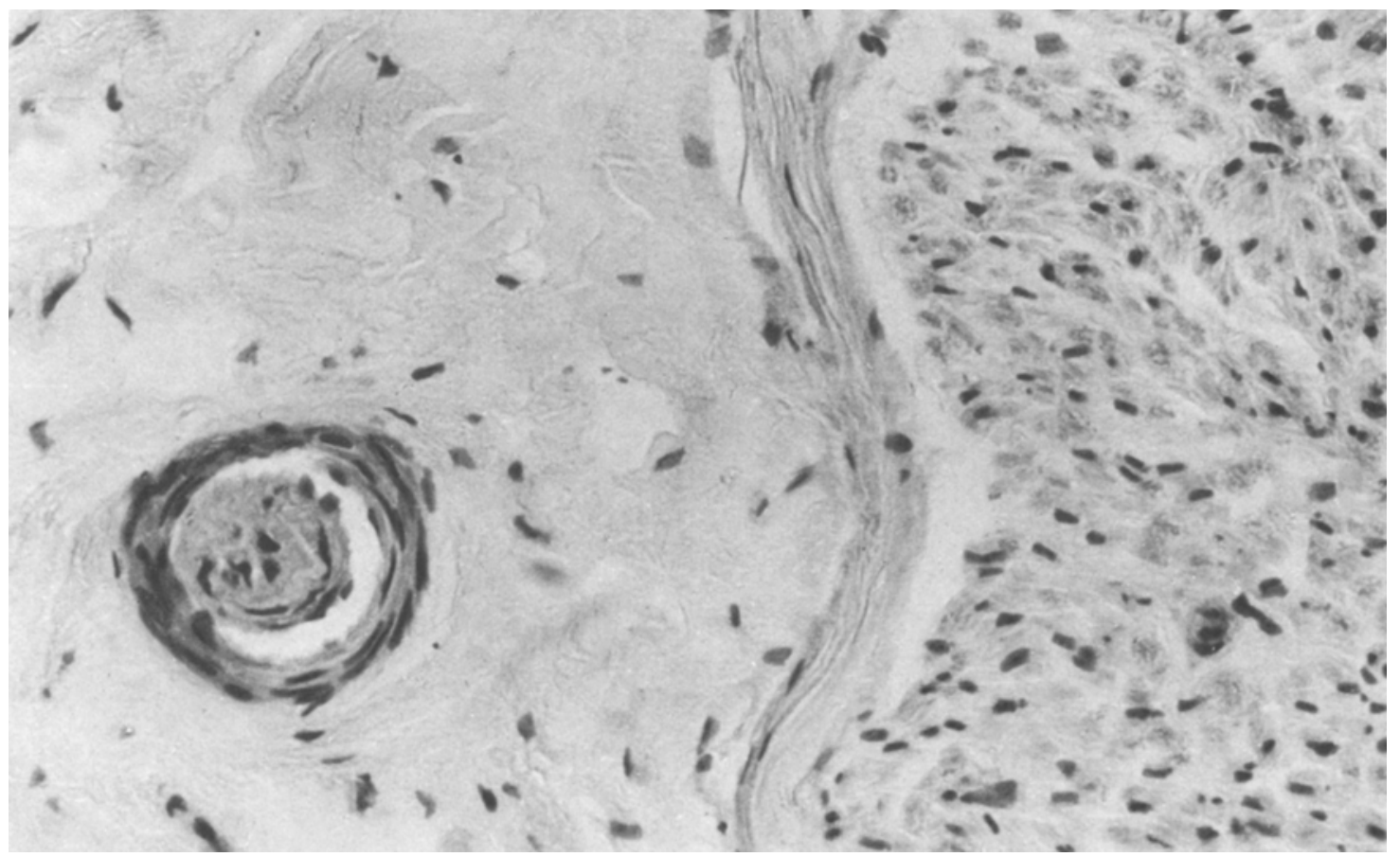

Fig. 4. Vessel containing rounded mass of thrombus. HE stain $\times 600$ 


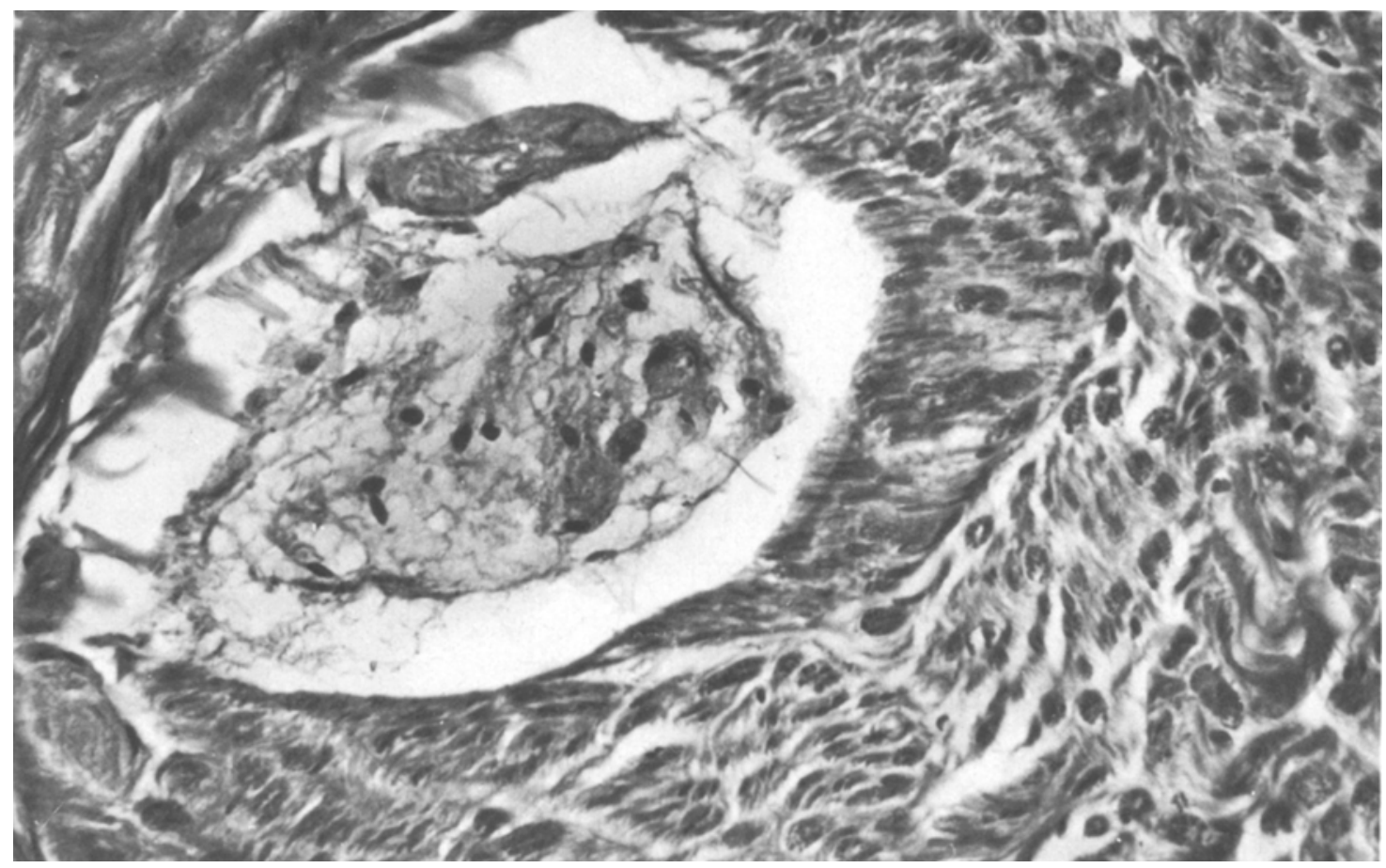

Fig. 5. Area of necrosis within a nerve bundle. HE stain $\times 600$

fibrin, and the appearances suggested that these were platelet aggregates.

In the case studied at post-mortem several small areas of haemorrhage were seen in the grey and white matter of the cerebral hemispheres and there was a moderate amount of blood in the subarachnoid space. No aneurysms or vascular malformations were found to account for the haemorrhage. Histological examination of the brain showed masses of fresh thrombus within cerebral vessels adjacent to areas of haemorrhage and within the thrombus large clumps of granular material were seen; the appearances suggested that these were platelet aggregates.

In only one case was there any evidence of minimal fibrin deposition in vessels from samples of skin removed at the time of biopsy and no evidence of fibrin deposition or older thrombus was found in any of the control specimens.

\section{Discussion}

During life there is a delicate balance between the deposition of fibrin on the vascular endothelium and its removal by fibrinolysis. Excessive deposition may result from abnormalities of the clotting system or defects in fibrinolysis. Abnormalities of both have been demonstrated in diabetics [7-9]. Neuropathic symptoms often vary in intensity and it may be that these relate to biochemical changes in the blood or nerve itself, or in changes in the vessel wall resulting in perfusion difficulties between the blood and neural tissues. In this study there was evidence of intravascular fibrin deposition in nine patients out of twentyfour in whom sural nerve biopsy was performed and in one case studied at post-mortem. In three cases fibrin was seen to be tracking through the endothelium into the surrounding wall. It was difficult to assess the role of platelet aggregates but in one biopsy clumps of granular material were present amongst the fibrin. In the autopsy case examination of fresh thrombus within cerebral and intraneural vessels showed clumps of granular material which were almost certainly platelets. It is of interest that no other tissue showed this abnormality. This case was important because it showed that intravascular coagulation occurs early in the course of diabetes, before clinical symptoms of neuropathy have appeared. The lesion does not appear to be secondary to degenerative vascular disease. Subsequently, in vitro studies of platelet aggregation in this group of patients with diabetic peripheral neuropathy have shown abnormalities of platelet function when compared to diabetics without neuropathy [11]. In four cases there was evidence of 
occlusion of vessels with older thrombus and in two of these areas of necrosis were seen in nerve bundles.

Many biopsies did not show evidence of fibrin deposition or thrombus formation. This is not surprising in view of the fact that a lesion anywhere along the length of a nerve fibre could interfere with its function and the deposition and clearing of fibrin would be expected to be phasic, in response to alterations in the metabolic environment. The chances of detecting significant lesions in such small biopsies would be expected to be small. In the cases showing evidence of thrombi in small vessels serial sections through the specimen rapidly passed through the affected area to a point at which the lumen was then patent.

The fact that vessels and skin removed at the time of biopsy showed no evidence of fibrin deposition, except in one case, could reflect on the different metabolic environment in this tissue or possibly on the fibrinolytic potential of small vessels in different tissues.

The "vascular theory" of diabetic neuropathy was proposed by Fagerberg [1], who described endothelial thickening and progressive sclerosis of small vessels in a large series of sural nerve biopsies. Examination of the colour photographs in Fagerberg's paper leads us to believe that some of the material staining with the Periodic-acid-Schiff reagent may be fibrin. In 1954 Anderson [12] postulated that the nodular lesion in diabetic glomerulo-sclerosis was the result of "deposition of fibrin or some other protein substance". Lendrum [13] and Lendrum et al. [14], also believed that the nodules were derived from fibrin which underwent a change to "pseudo-collagen" with time. Larsson [15] and Westberg and Michael [16] demonstrated fibrin, globulins and albumin in the glomerular basement membrane in diabetics. Farquhar [17] demonstrated fibrinogen and fibrin in the affected glomeruli by light and electron microscopy and immunofluorescence and postulated that the thickened basement membrane might arise from haematogenous elements - lipid, plasma, fibrin, haemosiderin, red cells, platelets and cholesterol crystals in the wall of retinal vessels.

A major objection to the "vascular theory" of diabetic nerve damage is based on the fact that clinical manifestations of diabetic neuropathy are frequently variable and recoverable. This is particularly true of the acute onset situation where severe motor neuropathy may recover relatively quickly, although less true in the more chronic, sensory type of neuropathy. Indeed many aspects of diabetic neuropathy are unlike other complications of diabetes in that the condition is not necessarily progressive or irreversible. Thus it has been difficult to accept the concept of "sclerotic" or degenerative small vessel disease as a cause of a condition which can improve so quickly. Rapid deterioration, followed by improvement in metabolic factors seemed a more logical explanation for this varying clinical situation. However, a disturbance of the delicate balance between the deposition and clearing of fibrin within vessels is an alternative explanation. Phasic activity is also explicable on the basis of re-canalisation of vessels occluded by thrombus or the establishment of anastomotic circulation pathways which might restore the normal myelinated state.

The results reported here indicate that the role of small vessel disease as a cause of nerve damage in the diabetic needs to be reassessed. It has been an assumption for many years that small vessel disease is a cause of diabetic complications in general, but the presence of fibrin deposition within nerve without similar changes in skin vessels suggest that more attention should be paid to the effect of the environment of a particular tissue on the vessels within it. With increasing understanding of factors which result in the deposition of fibrin within vessels it may be possible to interfere with these mechanisms in such a way that vascular occlusion does not occur..If such occlusion is important in the development of damage to or dysfunction of diabetic tissue it should be possible to prevent at least some complications independently of control of the blood sugar.

\section{References}

1. Fagerberg, S. E.: Diabetic neuropathy, a clinical and histological study on the significance of vascular affections. Acta med. scand. 345 (Suppl. 164), 1-97 (1959)

2. Thomas, P. K., Lascelles, R. G.: The Pathology of diabetic neuropathy. Quart. J. Med. 35, 489-509 (1966)

3. Greenbaum, D., Richardson, P. C., Salmon, M. V., Urich, H.: Pathological observations in six cases of diabetic neuropathy. Brain 87, 201-214 (1964)

4. Eliasson, S. G., Samet, J. M.: Alloxan induced neuropathies; Lipid changes in nerve and root fragments. Life Sci. 8, 493-498 (1969)

5. Pratt, J. H., Berry, J. F., Kaye, B.: Lipid class and fatty acid composition of rat brain and sciatic nerve in alloxan diabetes. Diabetes 18, 556-561 (1969)

6. Gabbay, K. H., O'Sullivan, J. B.: The sorbitol pathway. Enzyme localisation and content in normal and diabetic nerve and cord. Diabetes 17, 239-243 (1968)

7. Kwaan, H. C., Colwell, J. A., Suwanwela, N.: Increased platelet aggregation in diabetes mellitus. Diabetes 21, 844-845 (1972)

8. Fearnley, G. R., Chakrabarti, R., Avis, P. R.: Blood fibrinolytic activity in diabetes mellitus and its bearing on ischaemic heart disease and obesity. Brit. med. J. 1963 I, 921-923

9. Farid, N. R., Anderson, J. Martin, A., Weightman, D.: Fibrinolytic activity and treatment of diabetes. Lancet $1974 \mathrm{I}$, 631-632

10. Timperley, W. R., Preston, F. E., Ward, J. D.: Cerebral intravascular coagulation in diabetic Keto-acidosis. Lancet 1974 I, 952-956 
11. O'Malley, B. C., Timperley, W. R., Ward, J. D., Preston, F. E., Porter, N. R.: Platelet abnormalities in diabetic peripheral neuropathy. Lancet 1975 II, 1274-1278

12. Anderson, G. S.: The pathogenesis of diabetic glomerulosclerosis. J. Path. Bact. 67, 241-245 (1954)

13. Lendrum, A. C.: The validation of fibrin and its significance in the story of hyaline. In: Trends in clinical pathology (ed. G. Signy), pp. 160-187. London: British Medical Association 1969

14. Lendrum, A. C., Slidders, W., Fraser, D. S.: Renal hyaline. A study of amyloidosis and diabetic fibrinous vasculosis with new staining methods. J. clin. Path. 24, 373-396 (1972)

15. Larsson, O.: Studies of small vessels in patients with diabetes. Acta med. scand, 183 (Suppl. 480), (1967)

16. Westberg, N. G., Michael, A. F.: Immunohistopathology of diabetic glomerulosclerosis. Diabetes 21, 163-174 (1972)
17. Farquar, M. G.: Glomerular permeability investigated by electronmicroscopy. In: Small blood vessel involvement in diabetes mellitus (eds. M.D. Siperstein, A. R. Colwell, K. Meyer), pp. 235-243. Washington: American Institute of Biological Sciences 1964

Received: October 6, 1975, and in revised form: March 1, 1976

Dr. W. R. Timperley

Dept. of Medicine

The Royal Infirmary

Sheffield S 6 3DA

England 